Service social

\title{
Construire son identité de garçon : les représentations de la masculinité dans la littérature de jeunesse
}

\section{Anne-Marie Dionne}

Volume 58, numéro 1, 2012

URI : https://id.erudit.org/iderudit/1010443ar

DOI : https://doi.org/10.7202/1010443ar

Aller au sommaire du numéro

Éditeur(s)

École de service social de l’Université Laval

ISSN

1708-1734 (numérique)

Découvrir la revue

Citer cet article

Dionne, A.-M. (2012). Construire son identité de garçon : les représentations de la masculinité dans la littérature de jeunesse. Service social, 58(1), 85-98.

https://doi.org/10.7202/1010443ar
Résumé de l'article

En tant que produit culturel, la littérature de jeunesse véhicule des représentations à l'égard de la masculinité. Quelles sont donc ces représentations et comment influencent-elles la construction de l'identité sexuée des garçons ? Considérant que la littérature de jeunesse contribue de façon importante à la socialisation des enfants et des jeunes, il importe de connaître les normes sociales qu'elle laisse transparaître. Cet article aborde le sujet en considérant la primauté du genre masculin dans la littérature de jeunesse. Les représentations de la masculinité qui sont relevées sont analysées en fonction des théories qui s'inscrivent dans une perspective de construction sociale de l'identité sexuée. 


\title{
Construire son identité de garçon : les représentations de la masculinité dans la littérature de jeunesse
}

\author{
Anne-Marie Dionne
}

\section{RÉSUMÉ}

En tant que produit culturel, la littérature de jeunesse véhicule des représentations à l'égard de la masculinité. Quelles sont donc ces représentations et comment influencent-elles la construction de l'identité sexuée des garçons ? Considérant que la littérature de jeunesse contribue de façon importante à la socialisation des enfants et des jeunes, il importe de connaître les normes sociales qu'elle laisse transparaître. Cet article aborde le sujet en considérant la primauté du genre masculin dans la littérature de jeunesse. Les représentations de la masculinité qui sont relevées sont analysées en fonction des théories qui s'inscrivent dans une perspective de construction sociale de l'identité sexuée.

Mots-clés : Masculinités, littérature de jeunesse, identité sexuée, masculinité hégémonique.

\begin{abstract}
As a cultural product, children's literature conveys some representations regarding masculinity. What are those representations and how do they influence the development of boys' gender identity? Since children's literature contributes largely to the socialisation of youngsters and adolescents, it is important to recognize the social norms it transmits. This article addresses the topic by taking into account the primacy of masculine gender in children's literature. The representations of masculinity that are revealed are analysed in function of the theories which consider that gender identity is a social construct.
\end{abstract}

Key words: Masculinities, children's literature, gender identity, hegemonic masculinity.

Longtemps considérée comme une littérature de moindre importance, la littérature de jeunesse a depuis quelque temps acquis ses lettres de noblesse et elle est maintenant reconnue comme faisant partie intégrante du patrimoine culturel et littéraire au même titre que la littérature générale (Diament, 2004; Kalathakis, 2009). Que ce soit dans le milieu familial, dans le milieu scolaire ou au sein des organismes communautaires qui œuvrent auprès des familles ${ }^{1}$, les livres pour la jeunesse ont acquis un statut particulier. En effet, malgré la forte concurrence des autres formes de loisirs telles que le sport, la musique ou les jeux vidéo (Léon, 2004), le livre est considéré comme étant une valeur sûre par les intervenants qui se préoccupent du développement et du bien-être des enfants et des adolescents. Mais, comme le souligne

1. Tremblay (2003) fait état d'une multitude de projets centrés sur le livre jeunesse réalisés en milieux populaires par des organismes communautaires tels que les centres de la petite enfance (CPE) et autres services de garde, les centres locaux de services communautaires (CLSC), les directions de la santé publique (DSP), les bibliothèques publiques, l'éducation des adultes, l'éducation préscolaire, les groupes d'alphabétisation ou d'éducation populaire et les organismes communautaires de services à l'enfance et à la famille. 
Kalathakis (2009), les livres jeunesse font bien plus que de raconter des histoires, de divertir ou d'édifier les connaissances et la culture littéraire. Ils sont également des instruments qui transmettent aux enfants et aux adolescents des valeurs profondes concernant divers aspects de la société.

Au cours des dernières décennies, des recherches effectuées en grande partie dans des pays francophones et anglo-saxons ont mené à une prise de conscience que la littérature de jeunesse constitue un outil de socialisation qui est loin d'être anodin. Il a été démontré que cette littérature véhicule des valeurs et des idéologies qui s'expriment de façon explicite ou implicite à travers les textes et les images qui meublent l'univers littéraire dévolu aux jeunes lecteurs (Brugeilles, Cromer et Cromer, 2002; Cromer et Turin, 1998; Dafflon Novelle, 2006; Kolbe et Lavoie, 1981; Le Brun, 2002; Mallan, 2009; Weitzman, Eifler, Hokada et Ross, 1972). C'est le cas notamment des valeurs et des idéologies concernant les rôles sexués, les rapports sociaux entre les sexes, ou encore les représentations du genre féminin et du genre masculin. Dans une étude québécoise portant sur la construction sociale du féminin et du masculin, Descarries et Mathieu (2010, p. 60) notent, à propos de la littérature de jeunesse, que «force est de constater, qu'ici comme ailleurs, les livres d'aventure et d'action demeurent encore largement scénarisés autour de personnages masculins, alors que les contes de fées, remis à la mode dans une nouvelle mouture, continuent à pâtir des stéréotypes féminins ». Pour leur part, Johnston et Mangat (2002) mettent en évidence que les enfants et les adolescents sont exposés à une littérature écrite principalement par des hommes, dans laquelle les protagonistes sont principalement de sexe masculin.

Malgré cette prédominance masculine dans la littérature de jeunesse, on note que peu de chercheurs se sont penchés sur l'étude des masculinités qui s'y retrouvent. Comme le souligne Stephens (2002), sous l'influence du féminisme, les études de genres en littérature de jeunesse ont surtout mis l'accent sur les représentations féminines, ce qui n'est pas surprenant, étant donné l'impact du féminisme sur la culture et la littérature depuis la fin des années 1960. Toutefois, selon l'avis de Tremblay (2011), ce n'est pas parce que la masculinité ne suscite pas d'intérêt. En fait, depuis les années 1980, plusieurs chercheurs s'interrogent à ce sujet, comme le fait d'ailleurs de plus en plus fréquemment la culture populaire.

Dans le présent article, nous abordons le sujet en effectuant une recension des écrits sur les diverses représentations de la masculinité retrouvées dans la littérature de jeunesse. Mais auparavant, nous soulignons des éléments théoriques qui servent d'assises à l'étude de la construction de l'identité sexuée. Puisque la masculinité et la féminité sont deux concepts qui trouvent leur signification en étant mis en relation (Bourdieu, 1998), nous soulignons des asymétries à l'égard des genres masculins et féminins qui ont été observées dans les livres jeunesse. Par la suite, nous faisons état des représentations de la masculinité mises en évidence dans les recherches précitées. Pour faire le point, nous établissons des ancrages entre ces représentations de la masculinité et les éléments théoriques ayant été relevés. 


\section{La construction de l'identité sexuée}

Les théories qui visent à rendre compte de l'identité sexuée peuvent se regrouper selon différentes perspectives, allant de l'essentialisme pur à la construction sociale radicale, ce qui n'est pas sans susciter des débats opposant la nature et l'environnement (Demers, 2009). La perspective essentialiste évacue l'idée même du genre, favorisant plutôt des définitions rigides de la féminité et de la masculinité basées sur les différences biologiques, lesquelles moduleraient les comportements et les possibilités d'apprentissage de l'être humain. Ainsi, dès la naissance, selon qu'il soit un garçon ou une fille, l'enfant posséderait des attributs naturels, stables et immuables, assurant le développement d'une nature essentiellement masculine ou féminine (Baron-Cohen, 2003; Gurian, 1996). Une telle perspective ne fait pas l'unanimité dans l'espace intellectuel et depuis déjà plus de quarante ans, d'autres points de vue ont émergé. C'est le cas des perspectives se ralliant à la construction sociale et culturelle de l'identité sexuée, lesquelles mènent à percevoir les concepts de la féminité et de la masculinité dans une approche multidimensionnelle.

Lorsque l'identité sexuée est considérée dans une perspective de construction sociale, on met l'accent sur la notion du genre, laquelle se distingue clairement de la notion du sexe biologique. Oakley (1972, p. 16) établit la distinction suivante entre le sexe social et le sexe physiologique : «Sexe est un mot qui fait référence aux différences biologiques entre mâles et femelles [...] Genre est un terme qui renvoie à la culture : il concerne la classification sociale en masculin et féminin. » S'exprimant dans des termes analogues, Boisclair (2006) ajoute que si le sexe et le genre ont déjà été considérés comme étant deux dimensions indissociables l'une de l'autre (la masculinité étant associée aux hommes et la féminité étant associée aux femmes), les études ancrées dans les théories du genre ont permis de les détacher, permettant ainsi de mieux percevoir le caractère construit de l'identité sexuée.

L'étude de la masculinité est un sujet d'étude relativement nouveau qui s'inscrit dans les men's studies, un champ de recherche qui découle des mouvements féministes des années 1970. Les études s'intéressant à la masculinité considèrent des questions d'ordre social telles que les rôles des genres, les relations hommes-femmes, le patriarcat et la formation de l'identité masculine (Phillips, 2009). Au sujet de la formation de l'identité masculine, Connell (1995; 2000) démontre que la masculinité est le produit d'un apprentissage social et qu'il est maintenant convenu de parler des masculinités au pluriel. Pour lui, les masculinités se redéfinissent sans cesse, selon les contextes historiques et culturels. Or, comme il le souligne, une certaine forme de masculinité tend à s'imposer de façon plus manifeste que les autres. II s'agit de la masculinité hégémonique.

En introduisant le concept de la masculinité hégémonique, Connell (1987) souligne le caractère uniforme, fixe, restrictif et traditionnel de la masculinité qui habite l'imaginaire collectif de la société. Souvent considérée comme une norme sociale, la masculinité hégémonique domine les autres types de masculinité (les masculinités subordonnées) ainsi que l'ensemble des femmes. La masculinité hégémonique présente des traits qui se rapprochent des stéréotypes du « vrai » homme et servent d'assise au patriarcat. Selon Trujillo (2000), dans la société occidentale actuelle, ces principaux traits sont : l'expression et le contrôle du pouvoir et 
de la force physique, la réussite professionnelle, la hiérarchie familiale, le besoin de dépassement et l'hétérosexualité. Connell (2000) précise que la masculinité hégémonique est un idéal culturel qui reste assez éloigné de la majorité des hommes. D'ailleurs, les modèles qui représentent cet idéal sont souvent des personnages fantasmatiques, fabriqués par les médias et incarnés par des acteurs (par ex. John Wayne, Arnold Schwarzenegger), lesquels sont appelés à varier selon les lieux et les époques. II n'en demeure pas moins que l'influence de ces parangons de la masculinité se transpose dans l'idéologie populaire en ayant, par le fait même, une ascendance sur la construction de l'identité sexuée des enfants et des adolescents.

Des théoriciens de l'apprentissage social se sont peu à peu intéressés à la construction de l'identité sexuée de l'enfant et de l'adolescent. Deux dimensions particulières, soit l'influence de l'entourage social et la part active de l'enfant ou de l'adolescent dans la construction de ses connaissances sur les rôles sexués, sont prises en compte pour expliquer ce processus (Zaouche-Gaudron et Rouyer, 2002). D'après Bandura (1977; 1986) et Bussey et Bandura (1999), dès sa naissance, l'être humain construit son identité sexuée à travers le modelage et le renforcement. Le modelage lui permet d'acquérir des connaissances par le biais de médiateurs qui lui offrent des représentations sexuées du monde, que ce soit par le truchement des jeux, de la publicité, de la télévision ou des livres auxquels il a accès. Or, ces représentations sont modulées par les valeurs et les idéologies à l'égard des genres qui sont véhiculées dans la société, ce qui pourrait expliquer la prégnance et la ténacité de certains stéréotypes associés à la féminité et à la masculinité. Quant au renforcement, il prend en compte les réactions favorables ou non de l'entourage de l'enfant ou de l'adolescent lorsque ce dernier adopte des comportements conformes à son sexe ou typiques du sexe opposé. Encore là, l'entourage social, jugeant des conduites appropriées au sexe de l'enfant ou de l'adolescent, a le pouvoir de faire perdurer, d'atténuer ou de mettre un terme aux stéréotypes de genres.

Certains liens peuvent être établis entre la théorie de l'apprentissage social et les propos de Mosconi (2004) pour qui la construction de l'identité sexuée de l'être humain se forme graduellement, à travers ses expériences sociales avec les membres de son entourage et par son exposition aux médias, incluant la télévision et les livres. Dans son esprit en développement, les croyances et les valeurs dont l'enfant ou l'adolescent est témoin se transposent en une forme simplifiée des rôles et des attributs associés à masculinité et la féminité. Les représentations qu'il intègre progressivement dans ses schèmes de pensée sont susceptibles de s'organiser de façon dichotomique en ce qui concerne les caractéristiques physiques ou psychologiques de chacun des sexes, de même que les activités qu'il leur associe. En d'autres mots, l'enfant ou l'adolescent scrute le monde qui l'entoure afin de découvrir des règles auxquelles il ressent le besoin de se conformer. Ces règles deviennent les piliers sur lesquels repose la construction de son identité sexuée. De ce fait, survient alors le risque de perpétuer les stéréotypes de genres puisque, selon Mosconi (2004), l'environnement social valoriserait chez les garçons les expressions associées à la force, l'agressivité, le dynamisme et l'indépendance, alors que chez les filles, les expressions associées à la sociabilité et aux relations interpersonnelles seraient davantage encouragées. 
Selon Frawley (2008), les livres destinés aux enfants et aux adolescents contribuent également à former leurs premières perceptions concernant les genres. Ces perceptions sont d'autant plus durables et persistantes lorsque ces livres sont lus et relus à maintes reprises avec des personnes qui leur sont chères, telles que leurs parents ou leurs enseignants. L'auteur maintient que la façon dont les enfants et les adolescents perçoivent le monde qui les entoure et leur prise de conscience concernant la masculinité et la féminité sont grandement influencées par les images stéréotypées véhiculées par la littérature de jeunesse. Alors que les stéréotypes font partie des structures schématiques mentales qui les aident à organiser et comprendre leur environnement social pour le rendre prévisible et sécurisant (Bem, 1983), ils peuvent également avoir un impact important sur la façon dont ils perçoivent leurs rôles sociaux dans la vie. C'est pourquoi il convient de tenir compte des représentations des genres qui se manifestent dans la littérature de jeunesse.

\section{L'asymétrie des genres dans la littérature de jeunesse : la prédominance masculine}

Des recherches réalisées dans divers pays occidentaux démontrent que de façon générale, malgré quelques légères fluctuations au cours des ans, on retrouve des différences notables en ce qui a trait aux représentations du genre féminin et du genre masculin dans la littérature de jeunesse. Adoptant généralement l'analyse de contenu comme méthodologie, ces études constatent qu'à l'heure actuelle, la masculinité est prédominante dans l'univers littéraire destiné aux enfants et aux adolescents, que ce soit en Europe (Brugeilles, Cromer et Cromer, 2002), aux États-Unis (Weitzman et al., 1972) ou au Canada (Dionne, 2007; 2009). Par ailleurs, en plus de présenter des asymétries quantitatives importantes en ce qui concerne les représentations des genres masculins et féminins, les livres jeunesse véhiculent des stéréotypes sexistes flagrants vis-à-vis de la féminité et de la masculinité, ce qui peut avoir des conséquences importantes quant à la formation des schèmes mentaux des filles et des garçons concernant les rapports sociaux entre les genres (Frawley, 2008). Les études qui suivent démontrent que les représentations et les stéréotypes qui sont véhiculés par la littérature de jeunesse pourraient bien les mener à croire que dans la société, il est plus intéressant et valorisant d'être un garçon que d'être une fille.

Weitzman et al. (1972) sont considérés comme des précurseurs dans l'étude des stéréotypes de genres dans la littérature de jeunesse. En effectuant l'analyse d'un ensemble de livres primés par le prix Caldecott, le prix le plus prestigieux accordé aux œuvres de littérature pour la jeunesse publiées aux États-Unis, ils ont noté que les personnages masculins étaient représentés beaucoup plus fréquemment que les personnages féminins dans les livres analysés. De plus, les personnages féminins, représentés dans des rôles domestiques ou subordonnés, côtoyaient des personnages masculins qui apparaissaient comme des leaders remplissant des fonctions associées au pouvoir et à la réussite. À chaque décennie qui a suivi la réalisation de cette étude séminale, d'autres chercheurs (Crabb et Bielawski, 1994; Grauerholz et Pescosolido, 1989; Nilges et Spencer, 2002) ont corroboré les résultats obtenus par Weitzman et al. (1972), ce qui tend à démontrer que la situation, en ce qui concerne la répartition des rôles entre les personnages féminins et masculins, a peu évolué à travers les 
années et qu'à ce jour, elle est tout aussi inéquitable qu'il y a quarante ans. Comme le démontre l'étude qui suit, la situation est comparable de l'autre côté de l'Atlantique.

Selon Cromer (2010), le sexe et l'âge sont deux catégorisations sociales qui déterminent la majorité des personnages qui peuplent la littérature de jeunesse : ils sont soit de sexe féminin, soit de sexe masculin; soit adultes, soit enfants. En se référant aux résultats d'une étude d'envergure portant sur l'analyse de 537 albums illustrés publiés en France (Brugeilles, Cromer et Cromer, 2002), l'auteure met en évidence qu'il existe un déséquilibre notable entre le nombre de personnages masculins et le nombre de personnages féminins, qu'il s'agisse de protagonistes enfants ou de protagonistes adultes. De part et d'autre, le nombre de personnages masculins surpasse le nombre de personnages féminins. Selon Cromer, cet écart numérique entre les genres démontre incontestablement que «la primauté du masculin s'instaure »(p. 103). Ceci est d'autant plus vrai que les rôles occupés par les personnages dans le récit accroissent davantage les inégalités observées. En effet, il a été noté que les personnages féminins accèdent rarement aux rôles principaux, lesquels sont le plus souvent attribués aux personnages masculins, surtout lorsque les livres sont destinés aux enfants de 9 ans et moins. Non seulement les personnages masculins font plus souvent figure de héros, mais ils sont également plus nombreux parmi les simples figurants, ce qui fait en sorte que les personnages féminins sont largement éclipsés. Par ailleurs, dans la sphère publique encore plus que dans la sphère familiale, le personnage de sexe masculin décroche plus souvent le rôle de premier plan, ce qui dans l'ensemble laisse subtilement entendre que le garçon est l'acteur principal dans la société.

Peu d'études ont cherché à savoir comment se situe la littérature de jeunesse canadienne quant à la représentation des genres. Toutefois, deux études portant sur la littérature de jeunesse canadienne de langue française (Dionne 2007; 2009) nous ont permis de constater qu'à l'instar de la littérature de jeunesse américaine ou européenne, on y note des asymétries quantitatives importantes en ce qui concerne les représentations des personnages masculins et féminins. Dans l'une de ces études (Dionne, 2009), laquelle portait sur l'analyse des illustrations, il a été démontré que le genre féminin est sous-représenté dans les livres jeunesse, sauf dans les activités domestiques dans lesquelles on retrouve d'ailleurs très peu de personnages masculins. Par contre, en ce qui a trait aux activités personnelles et de loisir et aux activités professionnelles, les personnages masculins sont beaucoup plus présents que les personnages féminins. Une autre étude (Dionne, 2007) visait à faire l'analyse d'un ensemble de livres jeunesse afin d'y déceler les stéréotypes sexistes à l'égard des parents. Il s'est avéré que dans ces livres, on compte presque autant de pères que de mères. Cependant, les mères se distinguent nettement des pères par le fait qu'elles ont plus d'interactions avec leurs enfants, particulièrement en ce qui a trait aux soins qui leur sont prodigués. Quant aux pères, ils sont plus souvent que les mères engagés dans les jeux avec leurs enfants. Par ailleurs, cette étude a permis de constater qu'au cours des ans, l'image de la mère demeure traditionnelle, alors que l'image du père tend à s'enrichir quelque peu, bien qu'il se montre toujours moins engagé envers les enfants que ne l'est la mère. 
Les études présentées ci-dessus sont représentatives d'autres études sur le sujet (par ex. Alder, 1993; Anderson et Hamilton, 2005; Cromer et Turin, 1998; Dafflon Novelle, 2002; Détrez, 2010; Grauerholz et Pescosolido, 1989; Quinn et Flannery, 2006) qui, même si elles abordent différemment la question concernant les représentations des genres dans la littérature de jeunesse, abondent généralement dans le même sens. Nous nous limitons à celles qui sont déjà citées, car nous croyons qu'elles exposent clairement la situation. Dans l'ensemble, ces études, indépendamment de la langue ou du lieu géographique dans lesquels elles ont été réalisées, mettent en évidence que le genre masculin est largement plus représenté que le genre féminin dans l'univers littéraire des enfants et des adolescents. Alors que cet état de fait est maintenant bien établi, des questions s'imposent. Puisque la masculinité prédomine dans la littérature de jeunesse, comment est-elle représentée? Retrouve-t-on majoritairement la masculinité hégémonique, telle que décrite par Connell $(1987 ; 1995 ; 2000)$ ? Sinon, quels sont les autres types de masculinité que présentent les personnages masculins qui peuplent la littérature de jeunesse ? Les études dont il a été question jusqu'ici ne permettent pas de répondre à ces questions, mais de façon générale, elles laissent entrevoir que les rôles des personnages masculins sont stéréotypés, ce qui donne à penser que la masculinité hégémonique est souvent prédominante dans les livres jeunesse. Les questions mentionnées plus haut doivent être examinées quel que soit le sexe du lectorat concerné. En effet, tant les filles que les garçons peuvent être profondément influencés par les représentations de la masculinité dans la littérature de jeunesse. En présence de représentations stéréotypées de la masculinité, les garçons pourraient avoir tendance à croire que seule la masculinité hégémonique doit être valorisée et ils pourraient se sentir dévalorisés s'ils ne correspondent pas à cet idéal fantasmatique. De leur côté, les filles, devant les représentations récurrentes de la masculinité hégémonique, pourraient percevoir qu'il s'agit là du modèle masculin qui prédomine dans la société. Elles risquent également de croire qu'il est acceptable de rester cantonnées dans des rôles passifs, l'aventure et l'action étant réservées aux garçons. Les études dont il sera question dans la prochaine partie apportent quelques nuances en mettant en exergue que les masculinités s'expriment de façon légèrement différente selon l'âge du lectorat visé.

\section{Les représentations de la masculinité}

Malgré la prépondérance des personnages masculins dans la littérature de jeunesse, les études portant exclusivement sur les représentations de la masculinité semblent rares. L'étude de Le Brun (2002) fait exception. Pour dresser un portrait de la situation concernant les représentations de la masculinité dans les livres jeunesse, il a donc été nécessaire de recourir à des études s'intéressant de façon plus générale aux représentations des genres féminins et masculins dans ces œuvres littéraires. Nous avons choisi d'aborder la question des représentations de la masculinité selon le lectorat visé. Ainsi, on s'intéresse en premier lieu aux albums illustrés, ensuite, aux miniromans et enfin, aux romans pour adolescents. 


\section{L'album illustré}

L'étude de Brugeilles, Cromer et Cromer (2002) dont il a déjà été question permet de cerner certaines représentations de la masculinité dans les albums illustrés, ces ouvrages courts dans lesquels les images sont tout aussi importantes que les textes. Un examen des activités préférées des personnages a permis aux auteures de démontrer que vivre des aventures et faire des bêtises constituent les occupations principales des jeunes garçons. Quant aux hommes, ils s'adonnent au jardinage, bricolent, se reposent, lisent le journal, écoutent la radio ou la télévision. Quant à leurs activités professionnelles, ils sont aventuriers, médecins ou pharmaciens. Afin de mieux apprécier ces caractéristiques masculines, notons qu'en parallèle, les principales activités des filles se résument à se pomponner, se déguiser, faire le ménage et s'adonner à la danse. Quant aux femmes, elles sont essentiellement occupées aux tâches ménagères et sur le plan professionnel, elles sont caissières, institutrices ou mannequins.

D'autres études portant sur la représentation des genres dans les livres jeunesse (Hamilton, Anderson, Broaddus et Young, 2006; Marshall, 2004; Turner-Bowker, 1996) apportent des éléments additionnels pour constituer un portrait encore plus détaillé des représentations de la masculinité retrouvées dans les albums illustrés. Considérant l'ensemble de ces études, les principaux traits qui marquent les personnages masculins sont que de façon générale, ils sont indépendants, dominants et autoritaires. Ils sont surtout engagés dans des activités qui se réalisent dans des lieux publics ou dans la nature. En plus de vivre des aventures de tout genre, ils sont braves, héroïques, compétitifs et agressifs. À l'occasion, ils se portent au secours des filles ou des animaux. Enfin, ils aspirent à des professions socialement valorisées.

\section{Le miniroman}

Pour sa part, Le Brun (2002) s'est intéressée aux représentations de la masculinité dans la littérature de jeunesse en effectuant une analyse raffinée de tous les personnages principaux masculins de premier plan apparaissant dans un ensemble de 102 miniromans destinés aux enfants de 7 à 9 ans. Constitués en plusieurs séries, ces livres mettent en vedette 25 héros et 7 héroïnes. Le Brun est parvenue à relever sept ensembles de traits récurrents qui illustrent les représentations des héros masculins auxquels les jeunes lectrices et les jeunes lecteurs sont exposés. Ainsi, avec quelques légères variations, l'étiquette du jeune héros se détermine comme suit: il vit au sein d'une famille traditionnelle, entouré de parents aimants et de ses jeunes sœurs; il est amoureux, mais il sent qu'il doit cacher ce sentiment, ainsi que la jalousie qui l'accompagne; il rêve d'une amitié secrète ou d'une relation fusionnelle permettant de former une entité particulière (par ex. « amis à la vie, à la mort »); il a une relation privilégiée avec un animal, lequel est généralement de sexe féminin; il découvre les exigences de l'amitié, soit avec les filles ou les garçons; par le biais de la narration à la première personne, il se montre habile à décrire ses états psychologiques par l'introspection et la réflexion, ses préoccupations étant souvent d'ordre psychique ou familial; il surmonte ses peurs, que ce soit la peur de la violence, la peur de perdre un ami, la peur de perdre l'amour d'un parent, la peur du changement ou la peur de quitter le monde de l'enfance. 


\section{Le roman pour adolescent}

S'intéressant aux romans destinés aux adolescents, Détrez (2010) note qu'en ce qui concerne les best-sellers actuels de la littérature de jeunesse, les rôles féminins et masculins n'ont guère évolué au cours des dernières années. La répartition des rôles et des attributs reste très traditionnelle, surtout en ce qui a trait aux rôles parentaux. En ce qui concerne les héroïnes de ces romans, l'image de la jeune fille indépendante et autonome tente de s'imposer. Cependant, une lecture plus fine révèle une situation paradoxale. En effet, bien qu'elles « rêvent d'aventure [...], refusent le mariage et les tâches ménagères »(p. 76), elles n'échappent pas à une description traditionnelle et stéréotypée en ce qui concerne leurs caractéristiques physiques, vestimentaires et psychologiques. Quant à l'image des garçons, dans les romans analysés par l'auteure, l'image des adolescents demeure sans contredit très traditionnelle. Pour ces héros, pas question de se montrer sensible ou d'afficher sa peur. Les moments de doute envers leurs propres capacités sont rares et passagers. Dans les rapports intergénérationnels, les qualités qui leur sont imposées par leur père sont la bravoure et le courage. Néanmoins, l'auteure fait remarquer que ces valeureux héros se distinguent davantage par la ruse, l'intelligence et le courage plutôt que par la force et les muscles. II n'en demeure pas moins qu'ils n'ont pas le droit aux larmes, celles-ci étant réservées aux personnages secondaires.

\section{ConClusion}

À la suite des observations relevées dans les études concernant l'asymétrie des genres et plus particulièrement les représentations de la masculinité dans la littérature de jeunesse, deux constats s'imposent. Le premier est que les personnages de sexe masculin sont les principaux acteurs dans cet univers littéraire; le second, tout aussi clair, est que dans les livres destinés aux enfants et aux adolescents, il semble difficile de conjuguer la masculinité au pluriel. Bien au contraire, la masculinité hégémonique s'illustre pratiquement sans mélange. Si les asymétries au profit des personnages masculins ont déjà été soulignées (par ex. par Brugeilles, Cromer et Cromer, 2002; Weitzman et al., 1972), à notre connaissance, la représentation récurrente de la masculinité hégémonique dans les œuvres destinées à la jeunesse n’a jamais été dévoilée auparavant.

En tenant compte des divers lectorats visés, il est possible de déceler certaines tendances. Ainsi, il s'avère que dans les albums illustrés, la masculinité hégémonique règne sans partage. Les personnages masculins y présentent les traits distinctifs de la masculinité hégémonique mis en relief par Trujillo (2000). En ce qui concerne les miniromans, la situation se veut plus nuancée. Les jeunes héros gagnent en profondeur en étant souvent décrits comme des êtres sentimentaux et les relations humaines sont au cœur de plusieurs intrigues. Pourtant, les caractéristiques de la masculinité hégémonique ne manquent pas de s'illustrer de plusieurs façons : le jeune héros a des petites sœurs, ce qui lui permet de s'imposer dans la hiérarchie familiale; il est amoureux et jaloux, mais il cache ses sentiments; son animal de compagnie est généralement de sexe féminin; il éprouve des peurs, mais il réussit à les surmonter, ce qui dénote son besoin de dépassement. Enfin, dans les romans pour adolescents, on note que la masculinité hégémonique s'impose, tout autant que dans les albums illustrés, en présentant des héros qui se démarquent par un ensemble de valeurs traditionnellement masculines. Comme quoi l'adolescent, après avoir été provisoirement exposé à une conception légèrement plus 
ouverte de la masculinité, se doit, une fois l'adolescence venue, de rentrer dans les rangs en faisant siennes les conceptions traditionnelles de son sexe.

L'ensemble des résultats des recherches ayant été répertoriées dans cet article témoigne de l'urgence de sensibiliser les parents, les enseignants, les travailleurs sociaux, ainsi que les auteurs et les illustrateurs au fait que la littérature de jeunesse est un outil de construction sociale qui a le pouvoir de modeler les rapports sociaux entre les genres pour les générations futures. Le livre jeunesse jouit d'une autorité considérable auprès des enfants et des adolescents qui, tout en lisant individuellement ou en compagnie, vivent des aventures, éprouvent des émotions et partagent des jugements, portés par les images et les textes, qui sont le reflet d'idéologies données. Concernant les stéréotypes liés aux genres masculin ou féminin, il importe de les amener à faire une lecture critique des textes afin de remettre en question les discours dominants. À cet égard, les parents et les intervenants qui œuvrent auprès des enfants et des adolescents dans le milieu scolaire ou dans le milieu communautaire peuvent avoir beaucoup d'influence, car ils sont souvent les médiateurs entre le livre jeunesse et l'enfant ou l'adolescent. Le rapport de Tremblay (2003) illustre bien ce rôle important chez certains intervenants sociaux. L'auteure rapporte que dans le cadre d'un programme d'aide à l'éveil à la lecture et à l'écriture en milieux populaires, des intervenants responsables d'organismes tels que les CLSC, les CPE et les DSP ont mis sur pied des activités visant à promouvoir l'accès aux livres. Ces activités prenaient des formes variées : clubs de lecture, lectures animées dans les salles d'attente lors des séances de vaccination, expositions de livres, fêtes du livre, animations de la lecture dans des organismes s'adressant aux familles, etc. Ces quelques exemples démontrent bien que les intervenants des organismes mentionnés plus haut doivent être sensibilisés, au même titre que les parents et les enseignants, aux valeurs qui sont véhiculées dans les livres proposés aux enfants et à leur famille.

Selon Stephens (2002), l'oppression qui résulte du patriarcat et de la domination de la masculinité hégémonique demeure, encore aujourd'hui, un problème de justice sociale. Nous croyons que les idéologies auxquelles adhèrent les générations montantes peuvent contribuer à maintenir ou à défier les configurations de genres existantes. En les aidant à porter un regard critique sur les représentations de la masculinité qui se retrouvent dans la littérature de jeunesse, on peut amener les enfants et les adolescents à comprendre qu'il est possible de concevoir d'autres agencements des rôles dans lesquels les masculinités seraient valorisées dans leur pluralité, ce qui ne peut que contribuer à établir des rapports plus égalitaires entre les hommes et les femmes de demain.

Anne-Marie Dionne

Doctorat en éducation Ph. D. en psychopédagogie, Université Laval Professeure agrégée Université d'Ottawa Faculté d'éducation 


\section{BIBLIOGRAPHIE}

Alder, S. (1993). « Aprons and attitudes: A consideration of feminism in children's books », dans $\mathrm{H}$. Claire, J. Maybin et J. Swann (dir.), Equality matters: Case studies from the primary school, Clevedon (GB), Multilingual Matters, p. 111-123.

Anderson, D.A. et M. Hamilton (2005). «Gender role stereotyping of parents in children's picture books: The invisible father », Sex Roles, vol. 52, p. 145-151.

Bandura, A. (1977). Social learning theory, Englewood Cliffs (New Jersey), Prentice Hall.

Bandura, A. (1986). Social foundations of thought and action: A social cognitive theory, Englewood Cliffs (New Jersey), Prentice Hall.

Baron-Cohen, S. (2003). The essential difference: Men, women and the extreme male brain, Londres, Penguin.

Bem, L.S. (1983). « Gender schema theory and its implication for child development: Raising gender-aschematic children in gender-schematic society », Journal of Women in Culture and Society, vol. 8, n 4, p. 598-616.

Boisclair, I. (2006). «Les conceptions de l'identité sexuelle, le postmodernisme et les textes littéraires », Recherches féministes, vol. 19, n² 2, p. 5-27.

Bourdieu, P. (1998). La domination masculine, Paris, Le Seuil.

Brugeilles, C., I. Cromer et S. Cromer (2002). « Les représentations du masculin et du féminin dans les albums illustrés ou Comment la littérature enfantine contribue à élaborer le genre », Population, vol. 57, p. 261-292.

Bussey, K. et A. Bandura (1999). "Social cognitive theory of gender development and differentiation », Psychological Review, vol. 106, $\mathrm{n}^{\circ}$ 4, p. 676-713.

Connell, R.W. (1987). Gender and power: Society, the person and sexual politics, Cambridge, Polity Press.

Connell, R.W. (1995). Masculinities, Los Angeles, The University of California Press.

Connell, R.W. (2000). The Men and the Boys, St Leonards (AU), Allen \& Unwin.

Crabb, P.B. et D. Bielawski (1994). « The social representation of material culture and gender in children's books », Sex Roles, vol. 30, $\mathrm{n}^{\text {os }} 1-2$, p. 69-79.

Cromer, S. (2010). « Le masculin n'est pas un sexe : prémices du sujet neutre dans la presse et le théâtre pour enfants », Cahiers du Genre, $n^{\circ} 49$ : «Les objets de l'enfance », sous la dir. de S. Cromer, S. Dauphin et D. Naudier, Paris, L'Harmattan, p. 97-116.

Cromer, S. et A. Turin (1998). «Que racontent les albums illustrés pour enfants ? Ou comment présente-t-on les rapports hommes-femmes aux plus jeunes ? », Recherches féministes, vol. $11, \mathrm{n}^{0} 1$, p. 223-230. 
Dafflon Novelle, A. (2002). «La littérature enfantine francophone publiée en 1997. Inventaire des héros et héroïnes proposés aux enfants », Revue suisse des sciences de l'éducation, vol. $24, n^{\circ} 2$, p. 309-326.

Dafflon Novelle, A. (2006). «Identité sexuée: construction et processus », dans A. Dafflon Novelle (dir.), Filles-garçons : socialisation différenciée?, Grenoble, Presses universitaires de Grenoble.

Demers, V. (2009). La représentation de la masculinité dans les vidéoclips de musique populaire : le code visuel et l'expression de la vulnérabilité masculine, Mémoire de maîtrise, Montréal, Université de Montréal.

Descarries, F. et M. Mathieu (2010). Entre le rose et le bleu: stéréotypes sexuels et construction sociale du féminin et du masculin, Québec, Conseil du statut de la femme.

Détrez, C. (2010). «Les princes et princesses de la littérature adolescente aujourd'hui. Analyses et impressions de lecture », La lettre de l'enfance et de l'adolescence, vol. $4, \mathrm{n}^{\circ} 2$, p. 75-82.

Diament, N. (2004). «De la littérature de jeunesse considérée comme objet patrimonial », Bulletin des Bibliothèques de France (BBF), n 5 , p. 65-73.

Dionne, A.-M. (2007). «Étude des stéréotypes sexistes à l'égard des parents dans la littérature jeunesse canadienne-française », Revue de l'Université de Moncton, vol. 38, $\mathrm{n}^{\text {os }} 1-2$, p. 111143.

Dionne, A.-M. (2009). « La représentation des genres dans la littérature jeunesse : une analyse des illustrations des livres primés par les prix du Gouverneur général du Canada », Revue des sciences de l'éducation, vol. 35, $\mathrm{n}^{0} 2$, p. 155-175.

Frawley, T.J. (2008). «Gender schema and prejudicial recall: How children misremember, fabricate, and distort gendered picture book information », Journal of Research in Childhood Education, vol. 22, n 3, p. 291-303.

Grauerholz, E. et B.A. Pescosolido (1989). " Gender representation in children's literature: 1900-1984 », Gender \& Society, vol. 3, p. 113-125.

Gurian, M. (1996). The wonder of boys: What parents, mentors, and educators can do to shape boys into exceptional men, New York, Jeremy P. Tarcher/Putnam.

Hamilton, M.C., D. Anderson, M. Broaddus et K. Young (2006). « Gender stereotyping and under-representation of female character in 200 popular children's picture books: A twentyfirst century update », Sex Roles, vol. 55, p. 757-765.

Johnston, I. et J. Mangat (2002). «Making the invisible visible: Stereotypes of masculinity in canonized high school literature », dans J. Stephens (dir.), Ways of being male, New York, Routledge, p. 133-149.

Kalathakis, E. (2009). Littérature jeunesse : entre stéréotypes et ouverture : vers la construction d'un label culturel ?, Mémoire de maîtrise, Genève, Université de Genève. 
Kolbe, R. et J.C. Lavoie (1981). « Sex-role stereotyping in preschool children's picture books », Social Psychology Quarterly, vol. 44, n², p. 369-374.

Le Brun, C. (2002). «Les "Petits Hommes" du roman québécois pour lecteurs débutants », Littérature canadienne pour la jeunesse, $\mathrm{n}^{\text {os }} 105-106, \mathrm{p} .31-47$.

Léon, R. (2004). La littérature jeunesse à l'école : Pourquoi ? Comment?, Paris, Hachette.

Mallan, K. (2009). Gender dilemmas in children's fiction, Grande-Bretagne, Palgrave Macmillan.

Marshall, E. (2004). «Stripping for the wolf: Rethinking representations of gender in children's literature », Reading Research Quarterly, vol. 39, p. 256-270.

Mosconi, N. (2004). « De l'inégalité des sexes dans l'éducation familiale et scolaire », Diversité, vol. 138, p. $15-22$.

Nilges, L.M. et A.F. Spencer (2002). «The pictorial representation of gender and physical activity level in Caldecott medal winning children's literature (1940-1999): A relational analysis of physical culture », Sport, Education and Society, vol. 7, n ${ }^{0} 2$, p. 135-150.

Oakley, A. (1972). Sex, gender,society, New York, Harper Colophon Books.

Phillips, D. (2009). «Masculinity studies », dans J. O'Brien (dir.), Encyclopedia of gender and society, Californie, SAGE Publications, p. 512-516.

Quinn, S. et M. Flannery (2006). «Examining the culture of fatherhood in American children's literature: Presence, interactions, and nurturing behaviours of fathers in Caldecott award winning picture books (1938-2002) », Fathering: A Journal of Theory, Research, and Practice about Men as Fathers, vol. 4, p. 71-95.

Stephens, J. (2002). Ways of being male: Representing masculinities in children's literature and film, New York, Routledge.

Tremblay, H. (2003). Le plaisir de lire et d'écrire, ça commence bien avant l'école, Québec, Ministère de l'Éducation.

Tremblay, V.-L. (2011). Être ou ne pas être un homme: La masculinité dans le roman québécois, Ottawa, Les Éditions David.

Trujillo, N. (2000). « Hegemonic masculinity on the mound. Media representations of Nolan Ryan and American sports culture », dans S. Birrel et M. G. McDonald (dir.), Reading sport: Critical essays on power and representation, Boston, Northeastern University Press, p. 1439.

Turner-Bowker, D. (1996). «Gender stereotyped descriptors in children's picture books: Does "Curious Jane" exist in the literature ? », Sex Roles, vol. 35, p. 461-488.

Weitzman, L.J., D. Eifler, E. Hokada et C. Ross (1972). « Sex-role socialization in picture books for preschool children », American Journal of Sociology, vol. 77, nº 6, p. 1125-1149. 
Zaouche-Gaudron, C. et V. Rouyer (2002). «L'identité sexuée du jeune enfant : actualisation des modèles théoriques et analyse de la contribution paternelle », L'orientation scolaire et professionnelle, vol. $3, n^{\circ} 4$, p. 523-533. 